\title{
Analyses of melatonin, cytokines, and sleep in chronic renal failure
}

\author{
Aline Rodrigues Pinto ${ }^{1,2} \cdot$ Nathani Cristina da Silva ${ }^{2} \cdot$ Luciana Pinato $^{2}$
}

Received: 2 June 2015 / Revised: 14 July 2015 / Accepted: 4 August 2015 / Published online: 14 August 2015

(C) Springer-Verlag Berlin Heidelberg 2015

\begin{abstract}
Purpose Inflammation and oxidative stress are involved in the process of chronic renal failure (CRF). CRF patients show indication of sleep disturbances, and the melatonin rhythm, which modulates sleep, is abnormal in these patients; however, it is still unclear whether inflammation could be related to the blockage of melatonin production and sleep disturbances in this population. The aim of this study was to characterize and correlate sleep, the melatonin rhythm, and the levels of the inflammatory cytokines tumor necrosis factor (TNF) and interleukin (IL)-6 in patients with CRF and controls.

Methods Sleep was evaluated by the "Sleep Quality Index Pittsburgh" (PSQI) questionnaire, and melatonin and cytokine contents in saliva and blood samples, respectively, were analyzed by ELISA.

Results The CRF group scored higher on the global PSQI, which indicates a lower sleep quality and a higher prevalence of sleep disorders, than the control group. The CRF individuals also showed lower melatonin content than the control groups, both during the day and at night, and lacked rhythmicity in melatonin production. The CRF group also showed higher contents of TNF and IL- 6 than the control group and a negative correlation between TNF and melatonin content. Conclusion These results suggest that the sleep disorders observed in the CRF group were probably related to the low
\end{abstract}

Luciana Pinato

lpinato@marilia.unesp.br

1 Irmandade da Santa Casa de Misericórdia da cidade de Marília, Marilia, SP, Brazil

2 Department of Speech-Language and Hearing Therapy, São Paulo State University, Av. Vicente Ferreira, 1278, Marília, SP, Brazil CEP: 17515-901 production of melatonin observed in this population. The high level of TNF, as previously demonstrated in other pathologies, is probably involved in this blockage of melatonin production in $\mathrm{CRF}$.

Keywords Inflammation $\cdot$ Biological rhythms $\cdot$ Sleep quality $\cdot$ Immune-pineal axis $\cdot$ Behavior

\section{Introduction}

As a result of biochemical changes in glomerular filtration rate and/or parenchymal damage in patients with chronic renal failure (CRF), the kidneys of these patients produce inflammatory cytokines and hormones. Thus, inflammation and oxidative stress are involved in the process of CRF [1] and in the complications associated with it [2].

Accordingly, CRF patients tend to exhibit high plasma concentrations of inflammatory mediators, such as tumor necrosis factor (TNF), interleukins 1 and 6 (IL-1 and IL-6), and oxidative stress markers [3].

In addition to this inflammatory condition, CRF patients clinically present with a high prevalence of sleep disorders [4], the cause of which has been linked to a possible blockage of the nocturnal increase in melatonin content [5].

The pathway of melatonin synthesis involves the signaling of environmental light-dark alternation to the pineal gland. In the absence of light, serotonin is acetylated in the pineal gland by the action of arylalkyl-amine-N-acetyltransferase (AANAT), resulting in the precursor $\mathrm{N}$-acetylserotonin (NAS), which is then methylated by the action of acetylserotonin Omethyltransferase (ASMT) to generate 5-methoxy-Nacetyltriptamine or melatonin [6].

The physiological rise in melatonin secretion at night is well established, and the melatonin level peaks at 
approximately 2:00 a.m. [7]. Thus, under health conditions, the increase in nocturnal melatonin secretion and the release of melatonin into the bloodstream and cerebrospinal fluid indicate the darkness of the surroundings to the body. This makes melatonin a transducer of environmental photic information to the body and a modulator of several circadian rhythms, including the sleep-wake cycle, neuroendocrine responses, and body temperature [8], besides acting as an antiinflammatory molecule [9], a regulator of innate and acquired immune responses [10], a neuroprotective molecule, and a promoter of dendritic stability [11].

On the other hand, in pathological conditions, the production of melatonin can be altered. An unexplored hypothesis in CRF is the possible blockage of melatonin production by inflammatory molecules because the synthesis of this hormone by the pineal gland may be suppressed by pathogenassociated molecular patterns (PAMPs) and proinflammatory cytokines, as demonstrated previously in other conditions $[5,12]$.

Understanding the regulation and dynamics of melatonin synthesis in physiological and pathophysiological conditions is essential because of the wide range of beneficial actions of melatonin. Therefore, considering the possible correlation between the inflammatory condition, melatonin blockage, and sleep disturbances in CRF, the aim of this study was to characterize and correlate the biological variables of melatonin level, sleep parameters, and inflammatory cytokine levels in patients with CRF.

This pilot study provided subsidies to new studies that investigate pharmacological treatments to restore melatonin rhythm in order to improve sleep quality and avoid disturbances in several non-chronobiotic actions of this hormone.

\section{Methods}

\section{Participants}

This cross-sectional clinical study was in accordance with the Regulatory Norms on Human Research. The study protocol was approved by the ethics committee of the local institution (proc. $n^{\circ} 0667 / 2013$ ), and informed consent was obtained prior to the initiation of data collection.

The inclusion criterion in the CRF group was the diagnosis of CRF in hemodialysis treatment, and the exclusion criteria for both groups were the presence of neurological or psychiatric issues, and the use of psychoactive drugs, melatonin, or other medications that may influence melatonin synthesis and/ or release.

The study included 39 subjects between 29 and 79 years of age (54.9 \pm 3.3 years old; either gender), and they were divided into two groups - the CRF group $(n=20)$ and the control group $(n=19)$. The participants did not show clinical diagnosis for obesity or clinical history of obstructive sleep apnea syndrome (OSAS).

\section{Procedures}

All procedures in the CRF group were performed on the first day of hospitalization, before hemodialysis treatment.

The indicators of sleep disturbances were obtained from the Pittsburgh Sleep Quality Index (PSQI). The PSQI is a self-rated questionnaire that assesses sleep quality and disturbances over a 1-month time interval and probes clinically important and patient-relevant symptoms in the areas of sleep quality and quantity. The PSQI consists of 19 self-rated items divided into seven subscales: subjective sleep quality, sleep latency, sleep duration, habitual sleep efficiency, sleep disturbances, use of sleeping medications, and daytime dysfunction. Each subscale is rated from 0 to 3, with a higher score indicating a more severe sleep complaint. The summed score of the seven subscales yields a single global score that represents the patient's overall sleep experience; a lower total score reflects a better quality of sleep. A global PSQI score greater than 5 is an indication that the person has great difficulty in at least two components of sleep quality or moderate difficulties in more than three components of sleep quality [13].

\section{Saliva samples}

For quantification of melatonin, saliva samples were obtained in the home environment for the control group and in the hospital bed for the CRF group at six time points with intervals of $4 \mathrm{~h}$ over a period of $24 \mathrm{~h}$, beginning at 8:00 a.m. The patients received an individual labeled kit (Salivette, SARSTEDT, Germany) for collection and storage of saliva. For both groups, samplings at nighttime were conducted under low-intensity red light with the goal of not disturbing pineal synthesis of melatonin [14]. The presence of xerostomia in most of the CRF patients hampered the collection of saliva but did not make it impossible. The samples were transferred to the laboratory facility and stored at $-20{ }^{\circ} \mathrm{C}$ until processing. Salivary melatonin was quantified using a commercial ELISA kit according to the manufacturer's instructions (IBL, Hamburg, Germany), and the results are expressed in $\mathrm{pg} / \mathrm{mL}$. For statistical analysis, the accumulated day melatonin concentration (values at 8:00 a.m., 12:00 a.m. and 4:00 p.m.) was compared with the accumulated night melatonin concentration (values at 8:00 p.m., 00:00 a.m. and 04:00 a.m.).

\section{Blood samples}

For the CRF individuals, blood was collected with heparinized syringes (1:00 a.m. and 1:00 p.m.). The samples were transferred to the laboratory facility and centrifuged at 


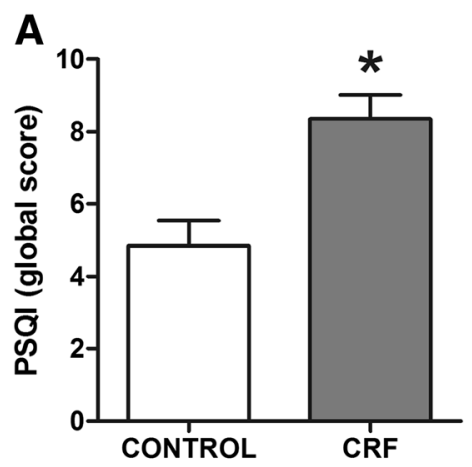

Fig. 1 Sleep quality in chronic renal failure and control subjects. a Mean \pm standard error of the mean of the overall score obtained by application of the Pittsburgh Sleep Quality Index in the control group $(n=19)$ and chronic renal failure group (CRF) $(n=20) . * P<0.05$. b Percentages $(\%)$ of individuals with and without sleep disorders obtained by application of

$1500 \mathrm{rpm}$ for $10 \mathrm{~min}$ at $4{ }^{\circ} \mathrm{C}$, and the supernatant was kept at $-80^{\circ} \mathrm{C}$ until processing. The TNF and IL- 6 contents in the plasma were determined using a commercial ELISA kit according to the manufacturer's instructions (IBL, Hamburg, Germany), and the results are expressed in $\mathrm{pg} / \mathrm{mL}$.

\section{Analysis of results}

Comparisons of the two groups, CRF and control, and of different phases (day and night) were performed using the Mann-Whitney $U$ test. Data were expressed as the means \pm standard error of the mean, and values were considered significantly different at $P<0.05$. The correlations between sleep disturbances and cytokine and melatonin levels and between cytokine and melatonin levels were examined using two-tailed Spearman coefficient computing $r$ for every pair of data sets. Statistical tests suitable for each analysis were performed with the statistical software Prism 6.0 for Windows (Graphpad Software, Inc.).

\section{Results}

The CRF group had a higher mean $(P=0.0001)$ global PSQI score $(8.3 \pm 3.0)$ than the control group $(4.8 \pm 3.0)$, which indicates worse sleep quality in the CRF group (Fig. 1a). In addition, the CRF group had a higher incidence of sleep disorders $(100 \%)$ than the control group (31.6\%; Fig. 1b).

The analysis of melatonin content in saliva showed that the patients in the CRF group had lower melatonin content than the control subjects, both during the day and at night (Fig. 2). During the day (light phase), the CRF subjects produced approximately $29 \%(0.7 \pm 0.1 \mathrm{pg} / \mathrm{ml})$ of the melatonin produced by the control subjects $(2.4 \pm 0.2 \mathrm{pg} / \mathrm{ml})$. At night (dark phase), the CRF subjects produced approximately $24 \%(1.1 \pm 0.2 \mathrm{pg} /$ $\mathrm{ml})$ of the melatonin produced by the control subjects $(4.6 \pm$ $0.6 \mathrm{pg} / \mathrm{ml})$.
In addition, the diurnal and nocturnal melatonin contents in the CRF group were not different, demonstrating the lack of rhythmicity in melatonin production in this group, unlike the control group, which showed normal rhythmicity, with melatonin content peaking at night (Fig. 2).

In regards to the levels of plasma inflammatory cytokines, the CRF group had a higher mean diurnal TNF level (62.3 \pm $9.2 \mathrm{pg} / \mathrm{ml})$ than the control group $(31.6 \pm 5.0 \mathrm{pg} / \mathrm{ml}$; Fig. 3a). Additionally, the CRF group had a higher mean diurnal IL-6 level $(34.4 \pm 16.6 \mathrm{pg} / \mathrm{ml})$ than the control group $(0.5 \pm 0.5 \mathrm{pg} /$ ml; Fig. 3b).

In the correlation analysis of concentrations of melatonin and cytokines (Figs. 4 and 5), a negative correlation was found between diurnal TNF concentration and nocturnal melatonin concentration (Fig. 4a). Although CRF patients with a high IL-6 concentration had low melatonin content, CRF subjects with a low IL-6 concentration also had low melatonin content, resulting in these two variables not having a statistical

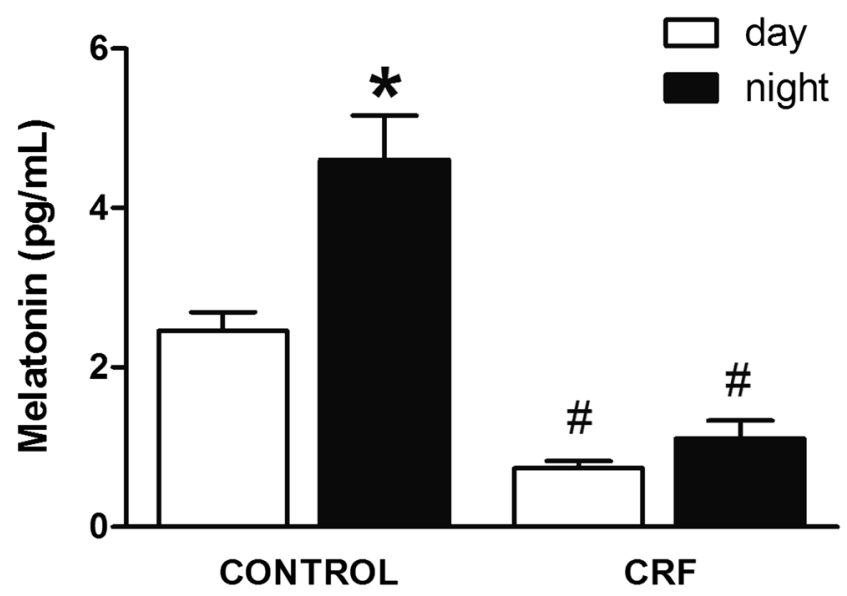

Fig. 2 Day/night salivary melatonin contents $(\mathrm{pg} / \mathrm{mL})$ in chronic renal failure and control subjects. Comparison between the mean \pm standard error of the mean of diurnal and nocturnal salivary melatonin contents between the control $(n=19)$ and chronic renal failure (CRF) groups $(n=$ 20), $* P<0.05$ 
Fig. 3 Plasma cytokines contents $(\mathrm{pg} / \mathrm{mL})$ in chronic renal failure and control subjects. a Comparison of diurnal TNF content (mean \pm standard error of the mean) between the control $(n=19)$ and chronic renal failure $(\mathrm{CRF})$ groups $(n=20)$. b Comparison of diurnal IL-6 content (mean \pm standard error of the mean) between the control $(n=$ $19)$ and chronic renal failure (CRF) groups $(n=20), * P<0.05$
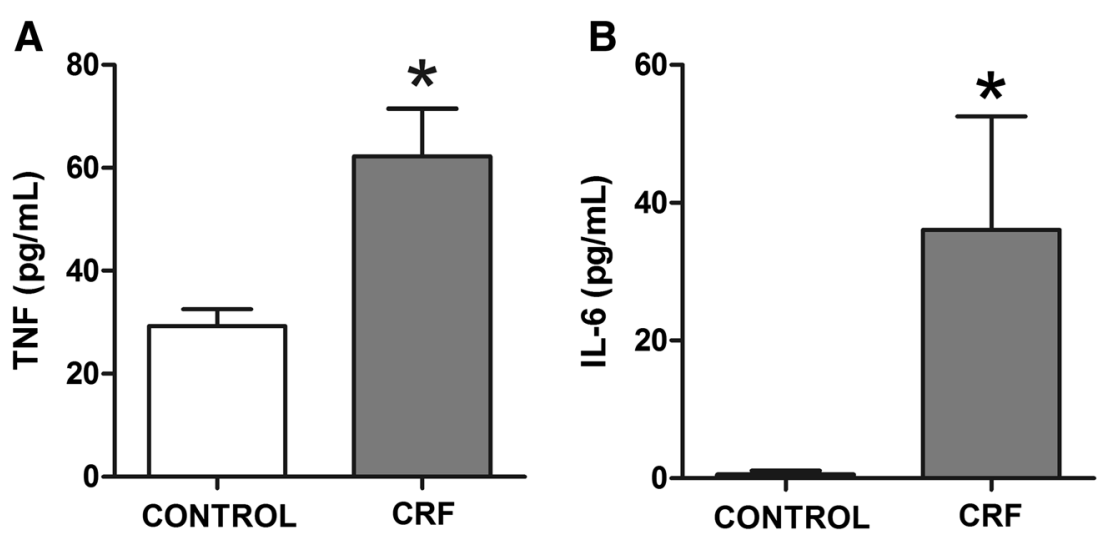

correlation (Fig. 5a, b). This was also true for nocturnal melatonin and nocturnal TNF concentrations (Fig. 4b).

\section{Discussion}

The present study found sleep disorders in $100 \%$ of patients with CRF, which is a higher incidence than in control subjects matched for age and sex (approximately $32 \%$ ) and is higher than previously reported prevalences of sleep disturbance in CRF patients, which have ranged from 50 to $80 \%[15,16]$. This high prevalence emphasizes the gravity of this complication of CRF as sleep quality impacts the vitality and morbidity and is crucial to the overall health of these patients $[15,17$, 18]. The importance of these findings is also supported by previous studies of other conditions, which demonstrated that sleep disorders result in daytime sleepiness and impairment in performance of various functions, including immune function and cardiovascular risk [19-21].

Although in the present study the presence of OSAS have not been investigated, the prevalence of this pathology is greater in CRF patients than in general population. Besides of this, the current literature suggests a bidirectional association between
CRF and OSAS through a number of potential pathological mechanisms. Following this idea, CRF may lead to OSAS by alterations in chemoreflex responsiveness, pharyngeal narrowing due to fluid overload, and accumulation of uremic toxins. On the other hand, OSAS can also accelerate loss of kidney function since chronic intermittent hypoxia directly or indirectly via renin-angiotensin system (RAS) activation [22] leads to kidney damage by facilitating glomerular hyperfiltration and systemic hypertension, both of which can impair kidney function [23].

The main sleep problems reported by this population included excessive daytime sleepiness [14], delayed sleep, fractionated sleep, waking up very early [24], nocturnal insomnia, increased sleep latency, and reduced sleep time [4].

The hypotheses of the causes of sleep disorders in previous studies of CRF include dialysis treatment [15] and the absence of the normal nocturnal increase in melatonin content $[4,5]$. Our results demonstrate a blockage in both diurnal and nocturnal production of melatonin in CRF, which generates a lack of rhythmicity in the content of this hormone. The salivary melatonin content of the control group at night during the beginning of sleep was $5.9 \mathrm{pg} / \mathrm{ml}$, which is in agreement with previous studies that reported a normal salivary melatonin content of $4 \mathrm{pg} / \mathrm{ml}$ for this period [25]. When salivary
Fig. 4 Correlation analysis between plasma cytokine TNF content $(\mathrm{pg} / \mathrm{mL})$ and salivary melatonin content $(\mathrm{pg} / \mathrm{mL})$. a Correlation analysis between nocturnal melatonin content and diurnal TNF content, $R=-0.457$ and $P<0.05$. b Correlation analysis between the nocturnal melatonin content and nocturnal TNF content, $R=-0.20$ and $P=$ 0.39 , both in the chronic renal failure group
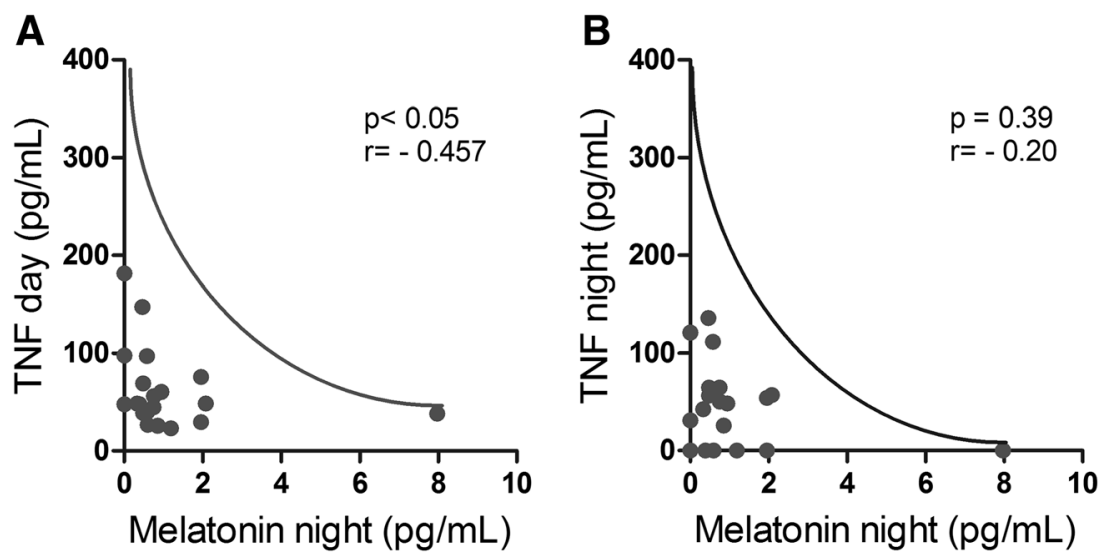
Fig. 5 Correlation analysis between plasma cytokine IL-6 content $(\mathrm{pg} / \mathrm{mL}$ and salivary melatonin content $(\mathrm{pg} / \mathrm{mL})$. a Correlation analysis between nocturnal melatonin content and diurnal IL- 6 content, $R=-0.20$ and $P=0.38$. b Correlation analysis between the nocturnal melatonin content and nocturnal IL- 6 content, $R=-0.08$ and $P=$ 0.72 , both in the chronic renal failure group
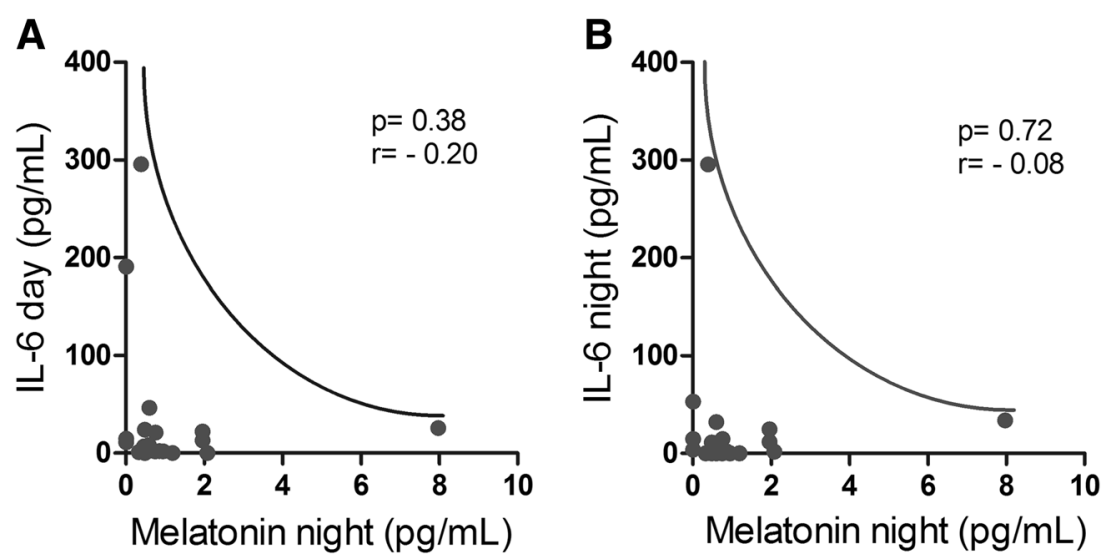

melatonin content is below this value and sleep latency is $>15$ min, patients are considered candidates for melatonin use to improve sleep parameters [17]. In this study, the CRF group showed $1.1 \mathrm{pg} / \mathrm{mL}$ melatonin during this period. Therefore, the use of melatonin in this group of CRF patients could be an option because hypnotics have frequently been prescribed in this population and have been found to be ineffective at improving sleep quality [5].

In addition to the chronobiotic function of melatonin, the results of this study suggest that it is necessary to consider other factors that are impacted by the blockage of melatonin production in CRF as melatonin is known to have functions in cellular processes, such as reduction of oxidative stress [10], inhibition of nuclear translocation of transcription factors [26], direct regulation of the action of several enzymes, intra-mitochondrial action, and regulation of apoptosis [27, 28]. Melatonin can act as an anti-inflammatory molecule [9], as a regulator of innate and acquired immune responses [10], metabolic functions, and several endocrine functions in peripheral tissues [29], as a neuroprotective molecule [12], and as a promoter of dendritic stability [11].

The results of the analysis of cytokine contents in the present study were in agreement with those of previous studies and demonstrated that patients with CRF tend to exhibit high plasma concentrations of the inflammatory mediators TNF and IL-6 [3].

Although other pathological models have shown that peripheral inflammation can block the production of melatonin in the pineal gland [5], the present study was the first to explore the hypothesis that the high levels of inflammatory cytokines in CRF patients could block the production of melatonin.

The present study demonstrated high diurnal TNF levels in CRF patients, and this was negatively correlated with the low nocturnal melatonin levels found in this population. Beside, this correlation do not means cause, this result suggest that TNF can be involved in the blockage of melatonin synthesis in the pineal gland in CRF as demonstrated previously in other conditions. This is evidence of the pineal-immune axis, which was previously demonstrated in other inflammatory conditions $[5,11]$. According to this concept, in pathological conditions, the synthesis and release of melatonin by the pineal gland become controlled by the immune system and, in turn, reciprocally influence the immune system. This concept involves an intricate and dynamic network that operates during the inflammatory response, integrating signaling pathways and regulation processes at the molecular, cellular, and organismic levels and resulting in the nocturnal peak of melatonin being suppressed in the early inflammatory response by the cytokine TNF $[5,30]$.

These findings open perspectives for the development of new therapies that can restore the physiological levels of melatonin and alleviate several problems related to lack of this hormone. These findings may also help improve sleep quality and, consequently, the quality of life of CRF patients.

In conclusion, individuals with CRF showed indications of sleep disorders, probably due to the low production of melatonin both during the day and at night and the lack of rhythmicity in melatonin production. In addition, high diurnal levels of TNF and IL-6 were observed in the CRF group compared to those of the control group, and a negative correlation between TNF level and nocturnal melatonin level was observed in the CRF group.

Acknowledgments This work was supported by grants from Fundação de Amparo a Pesquisa do Estado de São Paulo (FAPESP 11/51495-4) and CNPq (480208/2013-1).

Conflict of interest All authors certify that they have no affiliations with or involvement in any organization or entity with any financial interest (such as honoraria; educational grants; participation in speakers' bureaus; membership, employment, consultancies, stock ownership, or other equity interest; and expert testimony or patent-licensing arrangements), or non-financial interest (such as personal or professional 
relationships, affiliations, knowledge, or beliefs) in the subject matter or materials discussed in this manuscript.

Author contributions Conceived and designed the experiments: L.P. and A.R.P. Performed the experiments: A.R.P, N.C.S, and L.P. Analyzed the data: A.R.P, N.C.S, and L.P. Wrote the paper: L.P. and A.R.P.

\section{References}

1. Himmelfarb J (2004) Linking oxidative stress and inflammation in kidney disease: which is the chicken and which is the egg? Semin Dial 17:449-454

2. Cheung WW, Paik KH, Mak RH (2010) Inflammation and cachexia in chronic kidney disease. Pediatr Nephrol 25:711-724. doi:10. 1007/s00467-009-1427-z

3. Carrero JJ, Stenvinkel P (2010) Inflammation in end-stage renal disease - what have we learned in 10 years? Semin Dial 23:498509. doi:10.1111/j.1525-139X.2010.00784.x

4. Aperis G, Prakash P, Paliouras C, Papakonstantinou N, Alivanis P (2012) The role of melatonin in patients with chronic kidney disease undergoing haemodialysis. J Ren Care 38:86-92. doi:10.1111/ j.1755-6686.2012.00267.x

5. Koch BC, van der Putten K, Van Someren EJ, Wielders JP, Ter Wee PM, Nagtegaal JE, Gaillard CA (2010) Impairment of endogenous melatonin rhythm is related to the degree of chronic kidney disease (CREAM study). Nephrol Dial Transplant 25:513-519. doi:10. 1093/ndt/gfp493

6. Markus RP, Ferreira ZS, Fernandes PA, Cecon E (2007) The immune-pineal axis: a shuttle between endocrine and paracrine melatonin sources. Neuroimmunomodulation 14:126-133

7. Arendt J (1988) Melatonin. Clin Endocrinol (Oxf) 29:205-229. doi: 10.1111/j.1365-2265.1988.tb00263.x

8. Zhdanova IV, Lynch HJ, Wurtman RJ (1997) Melatonin: a sleeppromoting hormone. Sleep 20:899-907

9. Tomás-Zapico C, Coto-Montes A (2005) A proposed mechanism to explain the stimulatory effect of melatonin on antioxidative enzymes. J Pineal Res 39:99-104

10. Szczepanik M (2007) Melatonin and its influence on immune system. J Physiol Pharmacol 58:115-124

11. Reiter RJ, Paredes SD, Manchester LC, Tan DX (2009) Reducing oxidative/nitrosative stress: a newly-discovered genre for melatonin. Crit Rev Biochem Mol Biol 44:175-200. doi:10.1080/ 10409230903044914

12. Pinato L, da Silveira Cruz-Machado S, Franco DG, Campos LM, Cecon E, Fernandes PA, Bittencourt JC, Markus RP (2013) Selective protection of the cerebellum against intracerebroventricular LPS is mediated by local melatonin synthesis. Brain Struct Funct 220:827-840. doi:10.1007/s00429-013-0686-4

13. Bertolazi AN, Fagondes SC, Hoff LS, Dartora EG, Miozzo IC, de Barba ME, Barreto SS (2011) Validation of the Brazilian Portuguese version of the Pittsburgh Sleep Quality Index. Sleep Med 12:70-75. doi:10.1016/j.sleep.2010.04.020

14. Carazo I, Norambuena F, Oliveira C, Sánchez-Vázquez FJ, Duncan NJ (2013) The effect of night illumination, red and infrared light, on locomotor activity, behavior and melatonin of Senegalese sole (Solea senegalensis) broodstock. Physiol Behav 118:201-207. doi:10.1016/j.physbeh.2013.05.032

15. Parker KP (2003) Sleep disturbances in dialysis patients. Sleep Med Rev 7:131-143

16. Russcher M, Koch B, Nagtegaal E, van der Putten K, ter Wee P, Gaillard C (2012) The role of melatonin treatment in chronic kidney disease. Front Biosci (Landmark Ed) 17:2644-2656

17. Novak M, Shapiro CM, Mendelssohn D, Mucsi I (2006) Diagnosis and management of insomnia in dialysis patients. Semin Dial 19: 25-31

18. Koch BC, Nagtegaal JE, Kerkhof GA, ter Wee PM (2009) Circadian sleep-wake rhythm disturbances in end-stage renal disease. Nat Rev Nephrol 5:407-416. doi:10.1038/nrneph.2009.88

19. Bonnet MH (2005) Acute sleep deprivation. In: Kryger MH, Roth T, Dement WC (eds) Principles and practice of sleep medicine, 4th edn. Elsevier-Saunders, Philadelphia, pp 52-67

20. Krueger JM, Maide J (1990) A. Short analytical review: sleep as a host defense: its regulation by microbial products and cytokines. Clin Immunol Immunopathol 57:188-189

21. Wingard DL, Berkman LF (1983) Mortality risk associated with sleep patterns among adults. Sleep 6:102-107

22. Hostetter TH (2003) Prevention of the development and progression of renal disease. J Am Soc Nephrol 14:144-147

23. Ruggenenti P, Cravedi P, Remuzzi G (2012) Mechanisms and treatment of CKD. J Am Soc Nephrol 23:1917-1928. doi:10.1681/ ASN.2012040390

24. De Vecchi A, Finazzi S, Padalino R, Santagostino T, Bottaro E, Roma E, Bossi R (2000) Sleep disorders in peritoneal and haemodialysis patients as assessed by a self-administered questionnaire. Int J Artif Organs 23:237-242

25. Nagtegaal E, Peeters E, Swart W, Smits M, Kerkhof G, van der Meer G (1998) Correlation between concentrations of melatonin in saliva and serum in patients with delayed sleep phase syndrome. Ther Drug Monit 20:181-183

26. Cecon E, Fernandes PA, Pinato L, Ferreira ZS, Markus RP (2010) Daily variation of constitutively activated nuclear factor kappa $\mathrm{B}$ (NFKB) in rat pineal gland. Chronobiol Int 27:52-67. doi:10.3109/ 07420521003661615

27. Mayo JC, Sainz RM, Uria H, Antolin I, Esteban MM, Rodriguez C (1998) Melatonin prevents apoptosis induced by 6hydroxydopamine in neuronal cells: implications for Parkinson's disease. J Pineal Res 24:179-192

28. Burkhardt S, Reiter RJ, Tan DX, Hardeland R, Cabrera J, Karbownik M (2001) DNA oxidatively damaged by chromium(III) and $\mathrm{H}(2) \mathrm{O}(2)$ is protected by the antioxidants melatonin, N(1)-acetyl-N(2)-formyl-5-methoxykynuramine, resveratrol and uric acid. Int J Biochem Cell Biol 33:775-783

29. Cipolla-Neto J, Amaral FG, Afeche SC, Tan DX, Reiter RJ (2014) Melatonin, energy metabolism, and obesity: a review. J Pineal Res 56:371-381. doi:10.1111/jpi.12137

30. Carrillo-Vico A, Lardone PJ, Alvarez-Sánchez N, RodríguezRodríguez A, Guerrero JM (2013) Melatonin: buffering the immune system. Int J Mol Sci 14:8638-8683. doi:10.3390/ ijms 14048638 